\title{
Crizotinib in ROS1-positive non-small cell lung cancer: an Asian large phase II trial confirms its key-role
}

\author{
Paolo Maione, Cesare Gridelli \\ Division of Medical Oncology, S. G. Moscati Hospital, Avellino, Italy \\ Correspondence to: Cesare Gridelli. Division of Medical Oncology, S. G. Moscati Hospital, Contrada Amoretta, 83100 Avellino, Italy. \\ Email: cgridelli@libero.it. \\ Comment on: Wu YL, Yang JC, Kim DW, et al. Phase II Study of Crizotinib in East Asian Patients With ROS1-Positive Advanced Non-Small-Cell \\ Lung Cancer. J Clin Oncol 2018;36:1405-11.
}

Submitted Jul 31, 2018. Accepted for publication Aug 06, 2018.

doi: $10.21037 /$ tcr.2018.08.13

View this article at: http://dx.doi.org/10.21037/tcr.2018.08.13

The ROS1 oncogene encodes an orphan receptor tyrosine kinase related to anaplastic lymphoma kinase (ALK), along with members of the insulin-receptor family (1). ROS1 rearrangement can be found in several human cancers as cholangiocarcinoma, gastric cancer, ovarian cancer, glioblastoma multiforme, and with therapeutic implications in non-small cell lung cancer (NSCLC) (2-4). As a consequence of the rearrangement, a portion of ROS1, that includes the entire tyrosine kinase domain, is fused with 1 of 12 different pattern proteins (5). The ROS1 fusion kinases resulting from rearrangement are constitutively activated and drive cellular neoplastic transformation.

The incidence of ROS1 rearrangements in NSCLC is not high, resulting about $1 \%$, but if we considered only the never smoking population, it would be certainly higher (6). Clinical characteristics of patients with ROS1 rearrangements are similar to those of ALK positive patients (adenocarcinoma histology, younger age at diagnosis, never or light smokers). However, ROS1 rearrangements are mutually exclusive with ALK translocations as with EGFR, HER2, KRAS and BRAF mutations (7), but their prognostic role remains to be defined. Patterns of metastatic spread in ROS1 positive NSCLC are different from ALK-positive NSCLC. In fact at diagnosis ROS-positive patients have lower rates, compared with ALK-positive patients, of extrathoracic metastases (59\% versus $83 \%$ ) including brain metastases (19\% versus 39\%), but experience similar overall survival (3 versus 2.5 years) (6).

Importantly, ROS 1 is phylogenetically related to the ALK receptor tyrosine kinase. Thus, like ALK rearrangements in NSCLC, ROS1 fusions confer sensitivity to the ALK/ROS1/MET inhibitor crizotinib (7). Moreover, in preclinical models, crizotinib seems more effective in ROS1-positive than in ALK-positive NSCLC. However, crizotinib was originally developed as a MET inhibitor, and subsequently approved for ALK-positive NSCLC. A very relevant phase I study named PROFILE 1001 enrolled also 50 patients with advanced ROS1-positive NSCLC that were treated with crizotinib. The authors reported in this patient population a very high antitumor activity with an objective response rate (ORR) of $72 \%$ and a median progression-free survival (PFS) of 19.2 months (8). Also the median duration of response was relevant, resulting 17.6 months. Moreover, the authors did not report any correlations between the type of ROS1 rearrangement and the duration of treatment with crizotinib. Some retrospective analyses and prospective phase II trials reported similar response rates compared with PROFILE 1001, although shorter median PFS outcomes (range, 9.1-13.4 months) (9-12). Based upon this activity, the U.S. Food and Drug Administration, Europe and Asian countries expanded crizotinib's approval to patients with advanced ROS1-positive NSCLC, also without a randomized phase III trial because of the low incidence of this molecular subgroup of NSCLC.

Wu et al. very recently confirmed in a large prospective phase II, open-label, single-arm trial, the role of crizotinib in the treatment of ROS-1 positive advanced NSCLC (13). The authors enrolled, in East Asia, patients with advanced NSCLC harbouring a ROS1 rearrangement and that were pretreated with three or less lines of systemic therapies. 
In this trial ROS1 rearrangements were found through validated AmoyDx assay (Amoy Diagnostics, Xiamen, China). Crizotinib was administered orally at the dose of $250 \mathrm{mg}$ twice daily and continued until progression or unacceptable toxicity. The primary end point of the study was ORR, and in 16 months, 127 patients with ROS1positive NSCLC were enrolled at 37 sites in China, Japan, South Korea, and Taiwan. An ORR of 30\% was considered by the authors a clinically meaningful threshold for this study, and a lower limit of the two-sided $95 \%$ CI around the observed ORR greater than this threshold would have demonstrated the efficacy of crizotinib. The antitumor activity was clinically meaningful and consistent with previous reports. In fact the authors reported an ORR of 71.7\% (95\% CI, 63.0-79.3\%). Moreover, in 17 patients a complete response was reported, while the remaining 74 patients experienced a partial response. Thus, the primary end point ORR met the statistical prospectively defined criteria, and the positive treatment outcomes were reported both in patients with and without brain metastases and irrespective of the number of previous lines of systemic therapies. Moreover, treatment outcomes were also independent of enrollment country, age, sex, smoking status, or ECOG performance status. The onset of antitumor activity was also rapid, with a reported median time to response of 1.9 months (range, 1.6-15.8 months). Moreover responses were durable as expected from previous studies (median duration of response, 19.7 months; 95\% CI, 14.1 months to not reached). It is clinically relevant that among the 63 patients whose disease progressed during treatment with crizotinib, 43 (68.3\%) were treated beyond progression with crizotinib for 3 or more weeks (median duration, 20.7; range, 3.3-92.7 weeks). The other very important clinical outcome reported by $\mathrm{Wu}$ et al. for crizotinib in patients with ROS1-positive NSCLC was a long median PFS, resulted 15.9 months (95\% CI, 12.9-24.0 months). Moreover, it is to be considered that 45 (35.4\%) of 127 patients were still in follow-up for PFS at data cutoff. As stated before, clinical benefit was reported both for patients with $(n=23)$ and without $(n=104)$ baseline brain metastases, and median PFS resulted 10.2 months (95\% CI, 5.6-13.1 months) and 18.8 months (95\% CI, 13.1 months to not reached), respectively in the two patient subgroups. Although median survival was not a primary endpoint of this trial, the reported median overall survival of 32.5 months (95\% CI, 32.5 months to not reached) is very promising, also considering that $59.8 \%$ of patients were still in follow-up at data cutoff. This study is relevant considering that it is not only the first prospective phase II trial in East Asian patients but also the largest phase II trial of crizotinib in patients with advanced NSCLC harbouring a ROS1 rearrangement. The antitumor activity reported in this trial (ORR $71.7 \%$ ), is to be considered a clinically relevant outcome and similar to that reported by Shaw et al. in the PROFILE 1001 trial (69.8\%) (8) and to antitumor activity data from two prospective European phase II trials $(10,11)$. Also the timing of the response onset was early and similar in PROFILE 1001 and in the Asian study by $\mathrm{Wu}$ et al. Although the primary endpoint of the Asian trial was response rate, the result in terms of PFS is impressive (median, 15.9 months) and confirming the previous outcome reported in the PROFILE 1001 trial (19.2 months) (8). It is relevant that also Wu et al. reported clinical benefit achieved with crizotinib irrespective of the clinical characteristic of the patients as observed previously by other authors. In particular, antitumor activity was demonstrated independently of the number of previous lines of systemic therapies administered. Intracranial response was not assessed and this was considered also by the authors a limitation of this trial. Overall, the current results demonstrate that crizotinib is clinically effective and should be considered the standard of care for the treatment of patients with ROS1-positive NSCLC. In PROFILE 1001 , in 49 of 50 patients (98\%) ROS 1 rearrangement was identified using break-apart fluorescence in situ hybridization (FISH), while ROS1 fusion partners were identified with the use of next-generation sequencing or reverse transcriptase-polymerase chain reaction (RT-PCR) assays (8). Wu et al. assessed ROS1 rearrangements through an RT-PCR assay (AmoyDx) that is able to detect the majority of ROS1 rearrangements in lung cancer (14). The other widely used technique to detect ROS1 rearrangements and consequently to select patients to be treated with crizotinib is FISH. Another approach is to screen patients for ROS1 rearrangement by immunohistochemistry, as performed in the European trial, acsè (secured access to crizotinib for patients with tumors harboring a genomic alteration on one of the biological targets of the drug) (10). However, the AmoyDx RT-PCR diagnostic test used by Wu et al. in the Asian trial, guarantees a sensitivity of $100 \%$ and a specificity of $85 \%$ to $100 \%$ to detect ROS1 rearrangements when compared to FISH $(15,16)$. Thus, this test can be considered very effective to select patients with tumors harbouring ROS1 rearrangements and to be consequently treated with crizotinib. In terms of toxicity profile, Wu et al. did not report for crizotinib in this patient 
population unexpected toxicities $(8,17,18)$. Most adverse events reported by $\mathrm{Wu}$ et al. were mild, with no grade 5 treatment related adverse events observed, which indicates that the safety profile of crizotinib was generally good. Adverse events related to crizotinib treatment were easily managed by dosing interruptions and or reductions, and the rate of definitive treatment discontinuation due to toxicity was extremely low. Another relevant clinical outcome reported by Wu et al. was a trend toward an improvement in patient-reported global quality of life and reduction of lung cancer-related symptoms. Although the impressive treatment outcomes achieved with crizotinib in patients with ROS1-positive NSCLC, resistance to crizotinib finally occurs, as reported also by $\mathrm{Wu} e t$ al. Secondary mutations, epidermal growth factor receptor activation, and epithelialto mesenchymal transition are the main molecular mechanisms leading to resistance (19). Thus, although the excellent results achievable with crizotinib in this patient population, acquired resistance is a relevant clinical issue yet to be solved (6). Seven secondary ROS1 resistance mutations have been reported (19-21), and up-regulation of bypass signaling pathways (e.g., EGFR, RAS, and KIT) have also been observed (22).

Gainor et al. recently presented a large study on the mechanisms of resistance to crizotinib in patients with ROS1-positive NSCLC (6). The authors reported a secondary ROS1 resistance mutation as the main finding, especially when they considered the non-central nervous system (CNS) progressions during crizotinib treatment. Probably this reflects the low CNS penetration of crizotinib. Interestingly, a majority of these alterations were concentrated at one residue (G2032R). The frequency and spectrum of on-target mechanisms of crizotinib resistance in ALK- and ROS1-positive NSCLC is different. Indeed, ROS1-positive patients have a much higher frequency of on-target resistance mutations, but such mutations are concentrated in a narrower segment of the kinase, perhaps reflecting the greater activity of crizotinib against ROS1compared to ALK-positive NSCLC.

Data on cabozantinib, a multi-targeted TKI, and on lorlatinib, a next-generation ALK/ROS1 inhibitor have been accumulating, and clinical trials are ongoing, as potential drugs overcoming the resistance of ROS1-positive NSCLC to crizotinib $(23,24)$. In the next future, we can hope to have the possibility to administer sequentially more than one targeted agents against ROS1-positive NSCLC as we can already do for patients with ALK-positive NSCLC, in order to further improve the treatment outcomes in this patient population. In conclusion, $\mathrm{Wu}$ et al. have confirmed that crizotinib offers impressive treatment outcomes in terms of antitumor activity, quality of life, lung cancer symptoms control and safety profile in patients with ROS1positive advanced NSCLC, also in Asian patients, to the extent to be considered the standard first-line treatment in this molecular subgroup of NSCLC. Moreover, this Asian trial stimulates further prospective clinical trials with drugs aimed at overcoming resistance to crizotinib in this specific patient population.

\section{Acknowledgments}

Funding: None.

\section{Footnote}

Provenance and Peer Review: This article was commissioned and reviewed by the Section Editor Wei Xu (Division of Respiratory Disease, Department of Geriatrics, the First Affiliated Hospital of Nanjing Medical University, Nanjing, China).

Conflicts of Interest: Both authors have completed the ICMJE uniform disclosure form (available at http://dx.doi. org/10.21037/tcr.2018.08.13). The authors have no conflicts of interest to declare.

Ethical Statement: The authors are accountable for all aspects of the work in ensuring that questions related to the accuracy or integrity of any part of the work are appropriately investigated and resolved.

Open Access Statement: This is an Open Access article distributed in accordance with the Creative Commons Attribution-NonCommercial-NoDerivs 4.0 International License (CC BY-NC-ND 4.0), which permits the noncommercial replication and distribution of the article with the strict proviso that no changes or edits are made and the original work is properly cited (including links to both the formal publication through the relevant DOI and the license). See: https://creativecommons.org/licenses/by-nc-nd/4.0/.

\section{References}

1. Acquaviva J, Wong R, Charest A. The multifaceted roles of the receptor tyrosine kinase ROS in development and cancer. Biochim Biophys Acta 2009;1795:37-52. 
2. Rikova K, Guo A, Zeng Q, et al. Global survey of phosphotyrosine signaling identifies oncogenic kinases in lung cancer. Cell 2007;131:1190-203.

3. Lee J, Lee SE, Kang SY, et al. Identification of ROS1 rearrangement in gastric adenocarcinoma. Cancer 2013;119:1627-35.

4. Birch AH, Arcand SL, Oros KK, et al. Chromosome 3 anomalies investigated by genome wide SNP analysis of benign, low malignant potential and low grade ovarian serous tumours. PLoS One 2011;6:e28250.

5. Davies KD, Doebele RC. Molecular pathways: ROS1 fusion proteins in cancer. Clin Cancer Res 2013;19:4040-5.

6. Gainor JF, Tseng D, Yoda S, et al. Patterns of Metastatic Spread and Mechanisms of Resistance to Crizotinib in ROS1-Positive Non-Small-Cell Lung Cancer. JCO Precis Oncol 2017;2017. doi: 10.1200/PO.17.00063.

7. Bergethon K, Shaw AT, Ou SH, et al. ROS1 rearrangements define a unique molecular class of lung cancers. J Clin Oncol 2012;30:863-70.

8. Shaw AT, Ou SH, Bang YJ, et al. Crizotinib in ROS1rearranged non-small-cell lung cancer. $\mathrm{N}$ Engl J Med 2014;371:1963-71.

9. Mazières J, Zalcman G, Crinò L, et al. Crizotinib therapy for advanced lung adenocarcinoma and a ROS1 rearrangement: results from the EUROS1 cohort. J Clin Oncol 2015;33:992-9.

10. Moro-Sibilot D, Faivre L, Zalcman G, et al. Crizotinib in patients with advanced ROS1-rearranged non-small cell lung cancer (NSCLC). Preliminary results of the ACSe' phase II trial. J Clin Oncol 2015;33: abstr 8065.

11. Michels S, Gardizi M, Schmalz P, et al. EUCROSS: A European phase II trial of crizotinib in advanced adenocarcinoma of the lung harboring ROS1 rearrangements - preliminary results. J Thorac Oncol 2017;12: abstr S379-80.

12. Zhang L, Jiang T, Zhao C, et al. Efficacy of crizotinib and pemetrexed-based chemotherapy in Chinese NSCLC patients with ROS1 rearrangement. Oncotarget 2016;7:75145-54.

13. Wu YL, Yang JC, Kim DW, et al. Phase II Study of Crizotinib in East Asian Patients With ROS1-Positive Advanced Non-Small-Cell Lung Cancer. J Clin Oncol
2018;36:1405-11.

14. Uguen A, De Braekeleer M. ROS1 fusions in cancer: a review. Future Oncol 2016;12:1911-28.

15. Cao B, Wei P, Liu Z, et al. Detection of lung adenocarcinoma with ROS1 rearrangement by IHC, FISH, and RT-PCR and analysis of its clinicopathologic features. Onco Targets Ther 2015;9:131-8.

16. Shan L, Lian F, Guo L, et al. Detection of ROS1 gene rearrangement in lung adenocarcinoma: comparison of IHC, FISH and real-time RT-PCR. PLoS One 2015;10:e0120422.

17. Shaw AT, Reily GJ, Bang YJ, et al. Crizotinib in advanced ROS1-rearranged non-small cell lung cancer (NSCLC): Updated results from PROFILE 1001. Ann Oncol 2016;27:1206PD.

18. Camidge DR, Bang YJ, Kwak EL, et al. Activity and safety of crizotinib in patients with ALK-positive non-small-cell lung cancer: updated results from a phase 1 study. Lancet Oncol 2012;13:1011-9.

19. Song A, Kim TM, Kim DW, et al. Molecular Changes Associated with Acquired Resistance to Crizotinib in ROS1-Rearranged Non-Small Cell Lung Cancer. Clin Cancer Res 2015;21:2379-87.

20. Drilon A, Somwar R, Wagner JP, et al. A Novel CrizotinibResistant Solvent-Front Mutation Responsive to Cabozantinib Therapy in a Patient with ROS1-Rearranged Lung Cancer. Clin Cancer Res 2016;22:2351-8.

21. Facchinetti F, Loriot Y, Kuo MS, et al. Crizotinib-Resistant ROS1 Mutations Reveal a Predictive Kinase Inhibitor Sensitivity Model for ROS1- and ALK-Rearranged Lung Cancers. Clin Cancer Res 2016;22:5983-91.

22. Davies KD, Mahale S, Astling DP, et al. Resistance to ROS1 inhibition mediated by EGFR pathway activation in non-small cell lung cancer. PLoS One 2013;8:e82236.

23. Katayama R, Kobayashi Y, Friboulet L, et al. Cabozantinib overcomes crizotinib resistance in ROS1 fusion-positive cancer. Clin Cancer Res 2015;21:166-74.

24. Shaw AT, Felip E, Bauer TM, et al. Lorlatinib in nonsmall-cell lung cancer with ALK or ROS1 rearrangement: an international, multicentre, open-label, single-arm firstin-man phase 1 trial. Lancet Oncol 2017;18:1590-9.
Cite this article as: Maione P, Gridelli C. Crizotinib in ROS1positive non-small cell lung cancer: an Asian large phase II trial confirms its key-role. Transl Cancer Res 2018;7(Suppl 7):S792S795. doi: 10.21037/tcr.2018.08.13 